\title{
PREIM for nonlinear parabolic problems
}

\section{-edF}

\section{Amina Benaceur ${ }^{\star, \dagger}$, Alexandre Ern ${ }^{\star}$, Virginie Ehrlacher ${ }^{\star}$ and Sébastien Meunier ${ }^{\dagger}$}

${ }^{\star}$ CERMICS(ENPC) \& INRIA, France $\quad{ }^{\dagger}$ EDF R\&D, France

e-mail: amina.benaceurdenpc.fr

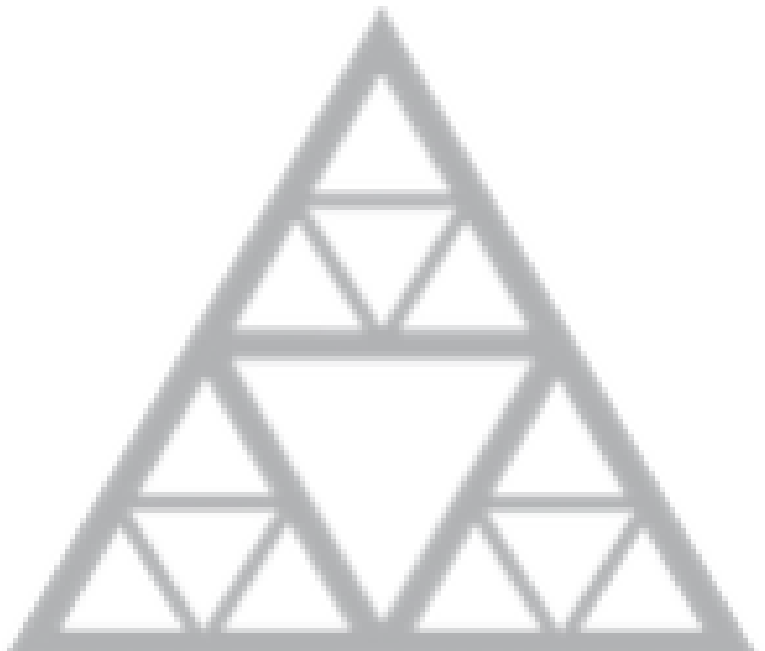

École des Ponts

ParisTech
The purpose of PREIM (Progressive RB-EIM) [1] is to reduce the offline costs of nonlinear parabolic reduced order models with accurate RB approximations in the online stage. The key idea is a progressive enrichment of both the EIM approximation and the RB space, in contrast to the standard approach where the EIM approximation and the RB space are built separately. PREIM uses highfidelity computations whenever available and RB computations otherwise. Another key feature of each PREIM iteration is to select twice the parameter in a greedy fashion, the second selection being made after computing the high-fidelity solution for the firstly selected value of the parameter.

\section{Model problem}

For $\mu \in \mathscr{P}^{\text {tr }}$, we consider the nonlinear heat transfer equation

$$
\left\{\begin{aligned}
\frac{\partial u_{\mu}}{\partial t}-\nabla \cdot\left(\left(\kappa_{0}+\Gamma\left(\mu, u_{\mu}\right)\right) \nabla u_{\mu}\right) & =f, & & \text { in }[0, T] \times \Omega, \\
-\left(\kappa_{0}+\Gamma\left(\mu, u_{\mu}\right)\right) \frac{\partial u_{\mu}}{\partial n} & =\phi_{e}, & & \text { on }[0, T] \times \partial \Omega, \\
u_{\mu}(t=0, \cdot) & =u_{0}(\cdot), & & \text { in } \Omega,
\end{aligned}\right.
$$

with the standard uniform ellipticity assumption $\beta_{1} \leq \kappa_{0}+\Gamma(\mu, z) \leq \beta_{2}$ and $0<\beta_{1}<\beta_{2}<\infty$.

\section{Standard RB-EIM approach}

For the RB approximation $\hat{\mathbf{u}}_{\mu}^{k}=\sum_{n=1}^{N} \hat{u}_{\mu, n}^{k} \theta_{n}$, the space-time discretized PDE (1) in reduced formulation reads

$$
\left(\mathbf{M}+\Delta t^{k} \mathbf{A}_{0}\right) \hat{\mathbf{u}}_{\mu}^{k}=\Delta t^{k} \mathbf{f}^{k}+\mathbf{M} \hat{\mathbf{u}}_{\mu}^{k-1}-\Delta t^{k} \mathbf{g}\left(\hat{\mathbf{u}}_{\mu}^{k-1}\right),
$$

with

$$
\mathbf{g}\left(\hat{\mathbf{u}}_{\mu}^{k-1}\right)=\left(\sum_{n=1}^{N} \hat{u}_{\mu, n}^{k-1} \int_{\Omega} \Gamma\left(\mu, \sum_{n^{\prime}=1}^{N} \hat{u}_{\mu, n^{\prime}}^{k-1} \theta_{n^{\prime}}\right) \nabla \theta_{n} \cdot \nabla \theta_{p}\right)_{1 \leq p \leq N}
$$

We introduce $\gamma(\mu, k, x):=\Gamma\left(\mu, u_{\mu}^{k}(x)\right)$ and seek a separated approximation of $\gamma$ in the form

$$
\gamma_{M}(\mu, k, x):=\sum_{j=1}^{M} \varphi_{\mu, j}^{k} q_{j}(x)
$$

In the standard RB-EIM, the EIM approximation (3) and the RB space $X_{N}=\operatorname{span}\left\{\left(\theta_{n}\right)_{1 \leq n \leq N}\right\}$ are built separately.

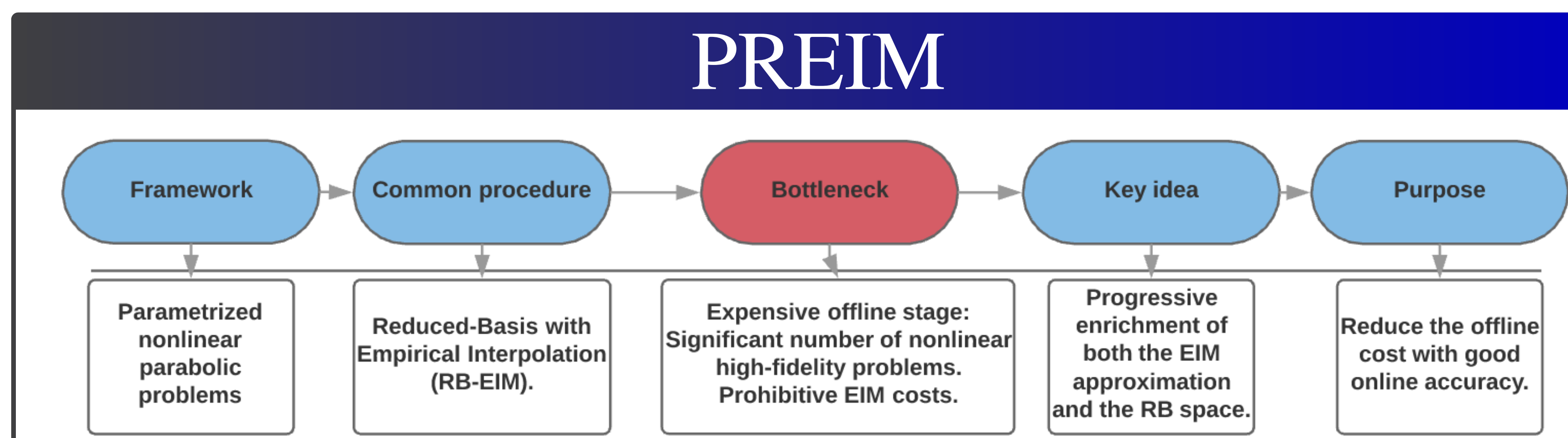

We introduce

$$
\bar{u}_{\mu}^{m, k}:= \begin{cases}u_{\mu}^{k} & \text { if } \mu \in \mathscr{P}_{m}^{\mathrm{HF}}, \\ \hat{u}_{\mu}^{m, k} & \text { otherwise }\end{cases}
$$

and the nonlinear function at iteration $m$

$$
\bar{\gamma}^{m}(\mu, k, x):=\Gamma\left(\mu, \bar{u}_{\mu}^{m, k}(x)\right) .
$$

PREIM has two goals:

- produce a set of RB functions $\left(\theta_{n}^{m}\right)_{1 \leq n \leq N^{m}}$;

- produce a rank- $m$ approximation of the nonlinearity (5) in the form

$$
\bar{\gamma}_{m}^{m}(\mu, k, x):=\sum_{j=1}^{m}\left(\bar{\varphi}^{m}\right)_{\mu, j}^{k} \bar{q}_{j}(x)
$$

three main ingredients:

- $\mathscr{P}_{m}^{\mathrm{HF}} \subset \mathscr{P}^{\mathrm{tr}}$ : Set that collects the previously computed HF trajectories

- $\mathscr{X}_{m}$ : Set of interpolation points $\left(\bar{x}_{i}\right)_{1 \leq i \leq m}$

- $\mathscr{Q}_{m}$ : Set of interpolation functions $\left(\bar{q}_{j}\right)_{1 \leq j \leq m}$

three main steps (the detailed algorithm appears in [1]):

- select a pair

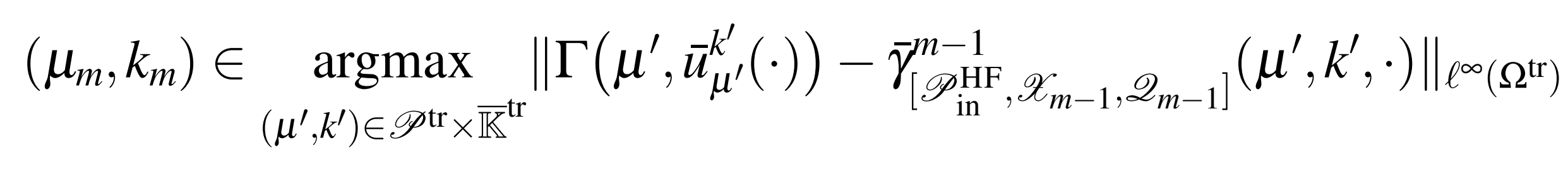

- compute $\mathscr{S}_{\text {out }}=\left(u_{\mu_{m}}^{k}\right)_{k \in \overline{\mathbb{K}}^{\mathrm{tr}}}$ and update the pair

$$
\left(\bar{\mu}_{m}, \bar{k}_{m}\right) \in \underset{\left(\mu^{\prime}, k^{\prime}\right) \in \mathscr{P} \text { out }}{\operatorname{argmax}} \underset{\mathbb{K}^{\mathrm{Hr}}}{\mathrm{Hr}}\left\|\Gamma\left(\mu^{\prime}, u_{\mu^{\prime}}^{k^{\prime}}(\cdot)\right)-\bar{\gamma}_{\left[\mathscr{P}_{\mathrm{in}}^{\mathrm{HF}}, \mathscr{X}_{m-1}, \mathscr{Q}_{m-1}\right]}^{\mathrm{H}}\left(\mu^{\prime}, k^{\prime}, \cdot\right)\right\|_{\ell^{\infty}\left(\Omega^{\mathrm{r}}\right)} .
$$

- update the reduced basis

$$
\Xi:=\operatorname{POD}\left(\tilde{\mathscr{S}}, \varepsilon_{\mathrm{POD}}\right)
$$

with $\Theta:=\left(\theta_{n}^{m}\right)_{1 \leq n \leq N^{m}}$ and $\tilde{\mathscr{S}}_{\text {out }}:=\left(u-\Pi_{\text {span }(\Theta)} u\right)_{u \in \mathscr{S}_{\text {out }}}$

and three accuracy criteria:

- $\varepsilon_{\mathrm{POD}}$ is the truncation threshold for the POD-based RB construction.

- $\varepsilon_{\mathrm{EIM}}$ is threshold for the approximation of the nonlinearity.

- $\varepsilon_{\mathrm{RB}}$ is threshold for the RB approximation.

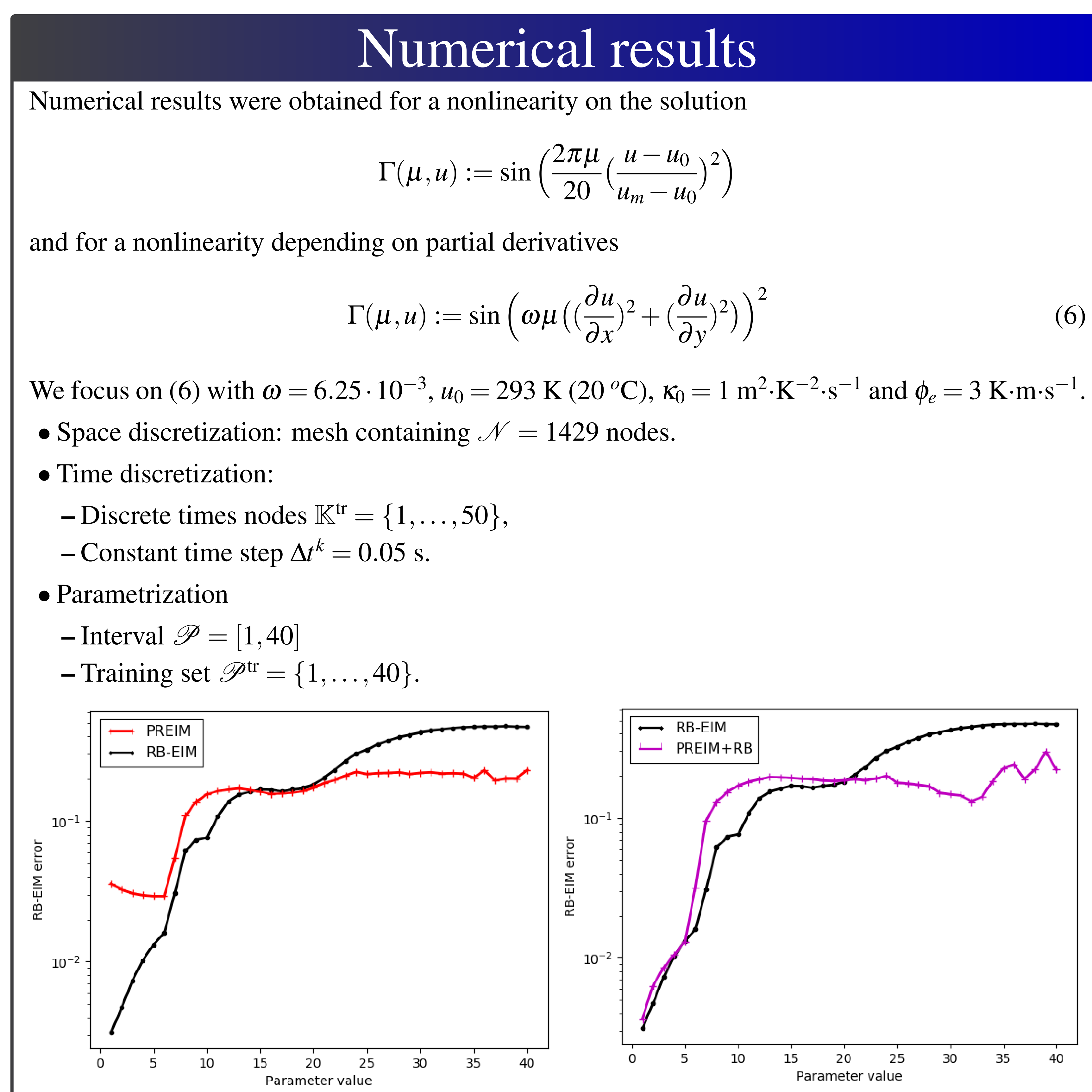

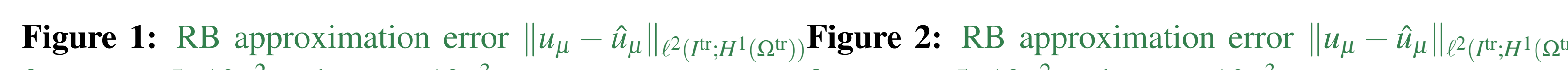
for $\varepsilon_{\mathrm{POD}}=5 \cdot 10^{-2}$ and $\varepsilon_{\mathrm{EIM}}=10^{-3} \quad$ for $\varepsilon_{\mathrm{POD}}=5 \cdot 10^{-2}$ and $\varepsilon_{\mathrm{EIM}}=10^{-3}$
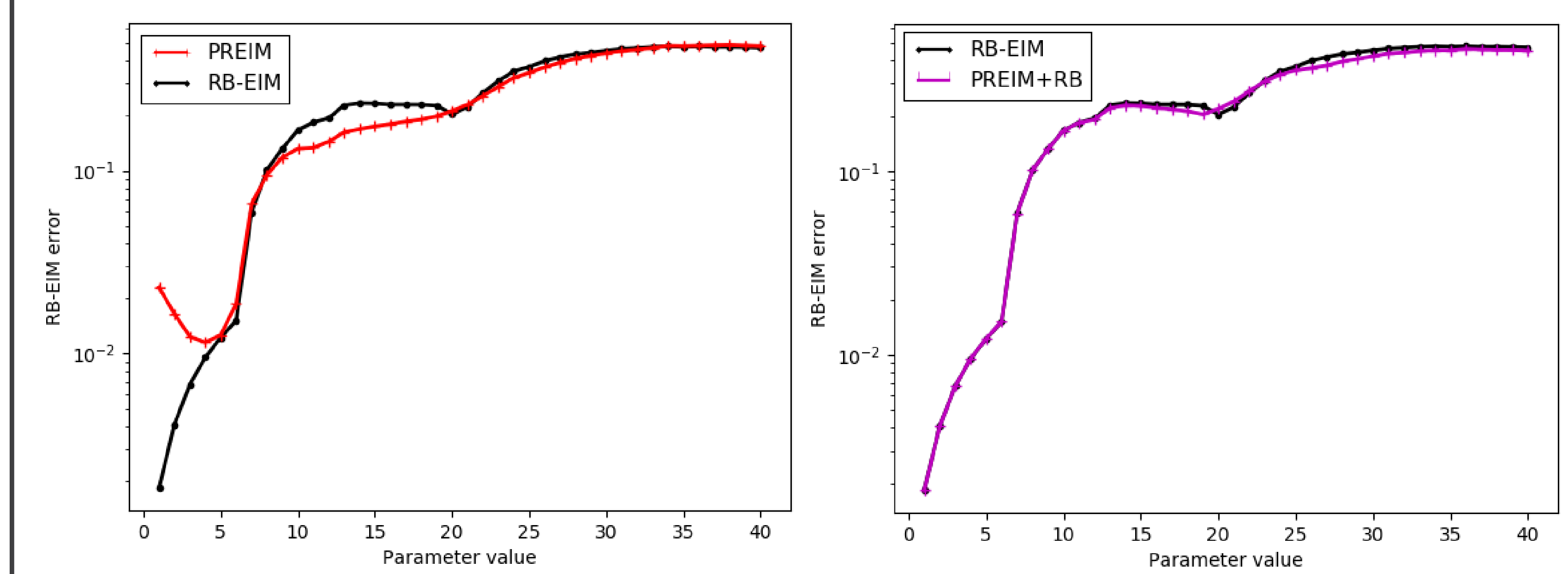

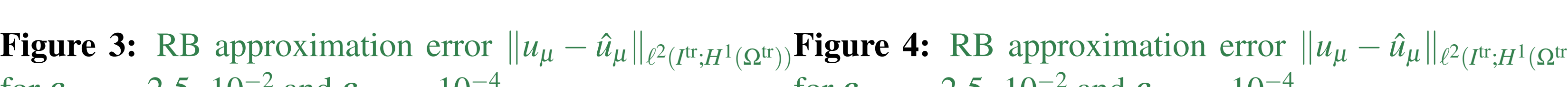
for $\varepsilon_{\mathrm{POD}}=2.5 \cdot 10^{-2}$ and $\varepsilon_{\mathrm{EIM}}=10^{-4} \quad$ for $\varepsilon_{\mathrm{POD}}=2.5 \cdot 10^{-2}$ and $\varepsilon_{\mathrm{EIM}}=10^{-4}$

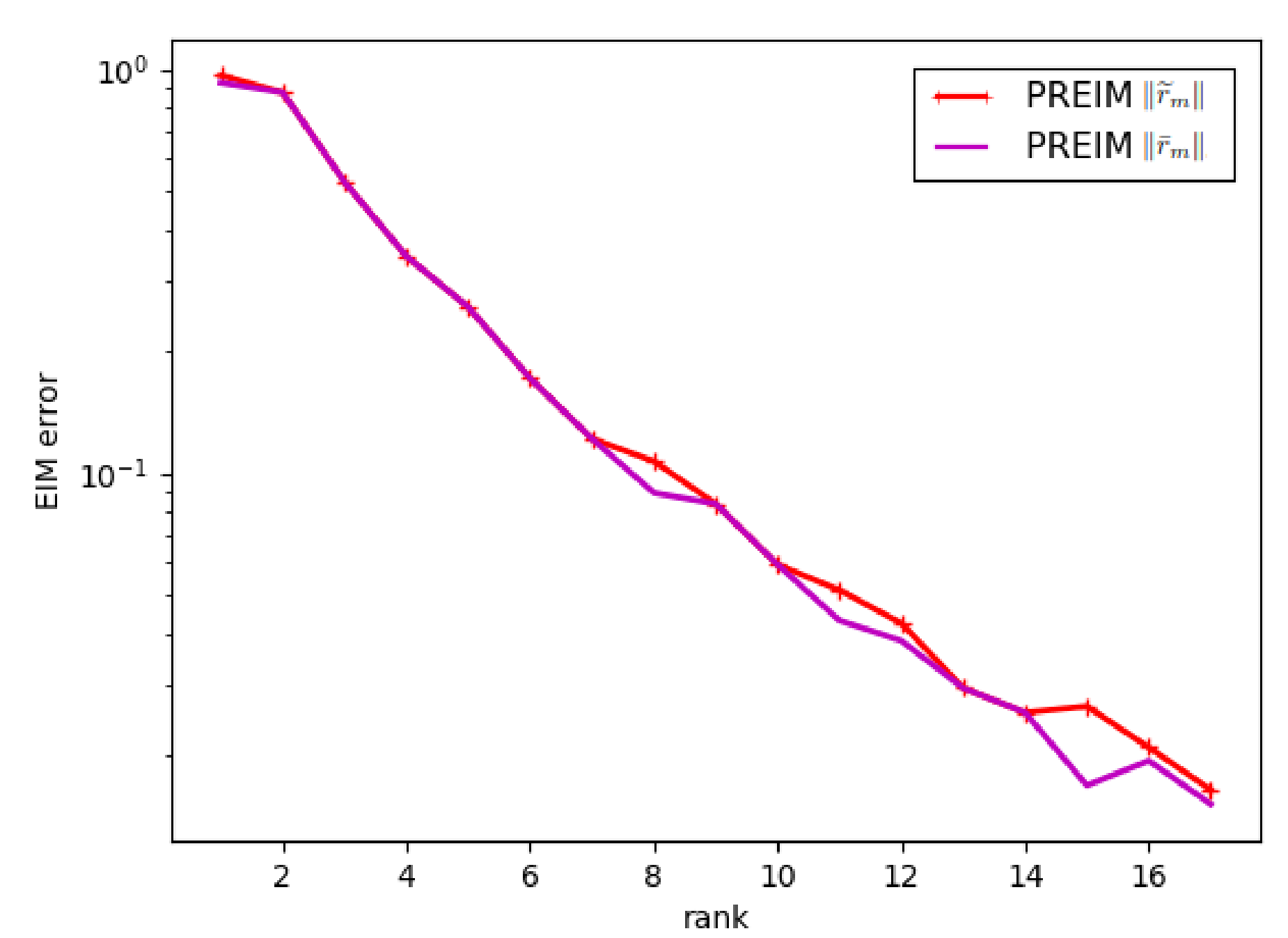

Figure 5: EIM approximation errors.

\begin{tabular}{|c||c|c|c|c|c|c|c|c|c|c|c|c|c|c|c|c|c|}
\hline$m$ & 1 & 2 & 3 & 4 & 5 & 6 & 7 & 8 & 9 & 10 & 11 & 12 & 13 & 14 & 15 & 16 & 17 \\
\hline \hline
\end{tabular} \begin{tabular}{|l||l|l|l|l|l|l|l|l|l|l|l|l|l|l|l|l|l|}
$\bar{\mu}$ & 21 & 21 & 21 & 8 & 21 & 21 & 21 & 8 & 9 & 21 & 9 & 21 & 9 & 9 & 9 & 6 & 21
\end{tabular} \begin{tabular}{|l|l|l|l|l|l|l|l|l|l|l|l|l|l|l|l|l|l}
\hline$\mu$ & 21 & 8 & 21 & 8 & 21 & 21 & 21 & 8 & 9 & 21 & 9 & 7 & 6 & 9 & 9 & 5 & 4 \\
\hline
\end{tabular}

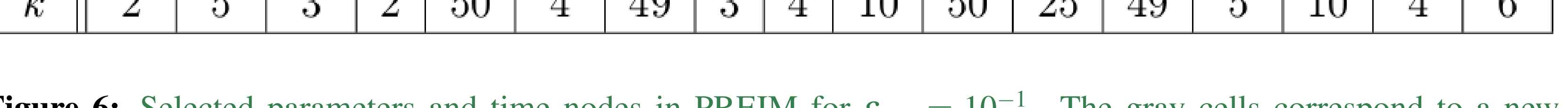

\section{Conclusions}

PREIM diminishes the offline expenses in the nonlinear RB method applied to unsteady nonlinear PDEs, as long as the computation of high-fidelity trajectories is the dominant part of the offline

\section{References}

[1] Benaceur, A., Ehrlacher, V., Ern, A., Meunier, S.: A progressive reduced basis/empirical interpolation method for nonlinear parabolic problems (2018). URL https://hal.archives-ouvertes.fr/hal01599304 